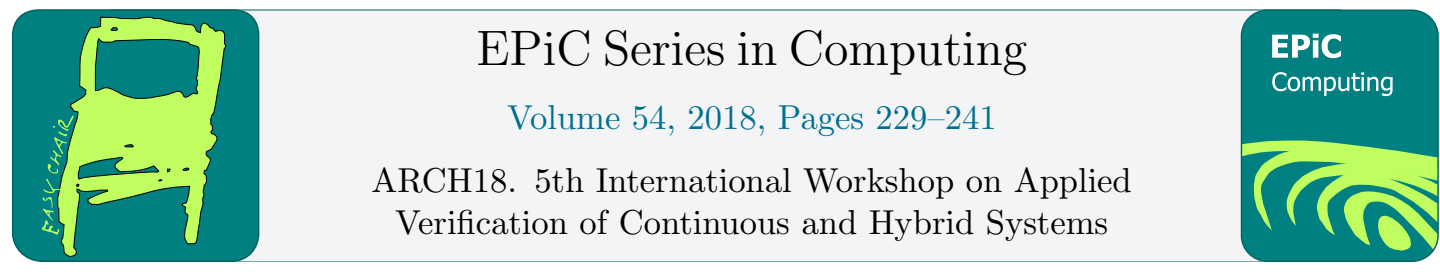

\title{
Lane change maneuver for autonomous vehicles* (Benchmark Proposal)
}

\author{
Nikolaos Kekatos ${ }^{1}$, Daniel Heß ${ }^{2}$, and Goran Frehse ${ }^{1}$ \\ 1 Verimag Lab, University of Grenoble Alpes, Grenoble, France \\ \{firstname.lastname\}@univ-grenoble-alpes.fr \\ 2 DLR, Braunschweig, Germany \\ daniel.hess@dlr.de
}

\begin{abstract}
Lane changes are known to be risky maneuvers both for autonomous vehicles and human drivers since they require changes in longitudinal and lateral velocities in the presence of other moving vehicles. In this paper, we propose a benchmark modeling a cooperative lane change maneuver that involves four fully autonomous vehicles; three in the left lane and one in the right. The vehicle driving in the right lane aims to move to the left lane while avoiding a collision with the other vehicles. Each vehicle is equipped with sensors and can also communicate with its neighboring vehicles. The vehicle dynamics are described by a dynamic bicycle model and each vehicle is equipped with a linear low-level controller that regulates its own longitudinal and lateral behavior. To guarantee that the maneuver is safe and the traffic rules are enforced, we employ a cooperative driving control scheme (in the spirit of supervisory logic) that decides the actions of each vehicle.
\end{abstract}

\section{Introduction}

The area of autonomous vehicles has attracted major research interest both from academia and industry [7]. By 2040, it is expected that autonomous vehicles will be the most common means of transportation [26]. An autonomous vehicle can take over the driving duties and has a big potential to reduce accidents caused by human errors, which account for $90 \%$ of the total accidents [27]. In this respect, automated driving is expected to entail safety and comfort as well as restrict accidents, crashes, and congestion [34]. However, the safe operation of vehicles in a dynamic environment must be guaranteed.

A particularly critical scenario concerns lane change maneuvers [3]. This maneuver is considered challenging as it involves changes in longitudinal and lateral speed and movement in the presence of other moving vehicles [3]. According to the National Highway Traffic Safety Administration, the main cause of lane change accidents is failure to detect the other vehicle and almost $80 \%$ of the accidents occur at speeds smaller than $25 \mathrm{~km} / \mathrm{h}[14]$.

*The authors gratefully acknowledge financial support by the European Commission project UnCoVerCPS under grant number 643921.

G. Frehse (ed.), ARCH18 (EPiC Series in Computing, vol. 54), pp. 229-241 


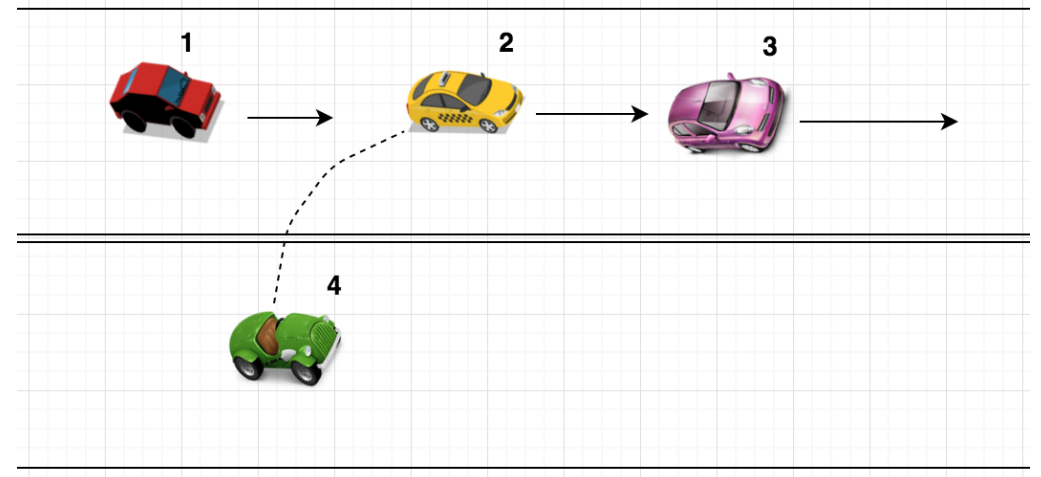

Figure 1: Lane Change Maneuver - Car in the right lane merging to the left lane.

The main components of a modern autonomous vehicle are localization, perception, and control [22]. The literature on vehicle path planning and control is rich, see the volumes [21, $30,19,5]$ and references therein. Control design of autonomous vehicles has been treated, e.g. in $[8,24]$. Different vehicle models for autonomous driving are presented in [22, 2]. Efficient trajectory planning with obstacle avoidance is a fundamental task for autonomous vehicles. The approaches can be divided into (i) planning in discrete space and (ii) planning in continuous space $[27,26]$. Very recently, an open source tool SPOT [23] was proposed to compute the future occupancy of multiple traffic participants on arbitrary road networks. A set of composable benchmarks for motion planning on roads can be found at [2].

Lane changes maneuvers can be divided on the basis of the existence of road infrastructure [18] and of a reference trajectory [28]. Predefined trajectories are known as motion primitives [9]. Motion primitives are computed offline and can be used to construct maneuver automata. Construction of formally verified maneuver automata using reachability analysis is investigated in $[1,16]$. Examples of control designs tailored to lane change maneuvers are tube-based MPC [11] and convex interpolation control [32]. As for metrics required to validate the suitability of a lane change maneuver, one can employ the time to collision, time headway, time to line crossing, or the inter-vehicle traffic gap [28, 13, 33].

In this paper, we propose a benchmark modeling a cooperative lane change maneuver that involves four autonomous cars; one in the right lane and three in the left lane. The vehicle in the right lane wants to make a lane change and moving behind the second vehicle and ahead of the third one. In the spirit of [28], the maneuver should be undertaken while (i) maintaining safety margins with all the surrounding vehicles, (ii) respecting the traffic rules, and (iii) satisfying the physical and design limitations.

\section{System Description}

In this section, we present the lane change scenario, the system specifications, the modeling part, and the control design. 
This benchmark presents a lane change maneuver involving four autonomous vehicles (see Figure 1). We consider that there are three vehicles in the left lane that have already formed a platoon and they move with the same speed. The desired speed is assumed to be predefined and is an external (constant) input to our system. We also assume that the maneuver has already been requested, e.g. by a higher layer (road infrastructure) or due to an emergency (obstacle avoidance). The vehicle in the right lane has to change its lane and merge to the left one. We consider that the vehicle should move in between two specific vehicles, i.e. tail (no. 1) and interior (no. 2). This maneuver can be seen as an automated merging maneuver [25], i.e. how to insert a vehicle from on-ramp in the middle between two pre-selected vehicles of a platoon in the main lane.

The lane change maneuver consists of two phases. The first phase is the preparation of the maneuver and the second is the maneuver itself. Once the maneuver is completed, the platooning formation should be achieved. In other words, the desired inter-vehicle distances and speeds should be reached.

We assume that the vehicles are equipped with orientation, position, and velocity sensors; thus, all the state variables can be measured. Also, the vehicles can communicate their longitudinal position and speed with their neighboring vehicles.

\section{$2.1 \quad$ Specifications}

The specs that the vehicle platoon should satisfy are taken from [28, 15]. Each vehicle should

1. maintain safety margins with all the surrounding vehicles,

2. respect the traffic rules, and

3. satisfy physical and design limitations.

More precisely, these specifications can be expressed as

1a. the distance of two neighboring vehicles should never be smaller than a given threshold ${ }^{1}$,

1b. the vehicles of the platoon should maintain a constant time gap $\left(t_{\text {gap }}\right)$ between each other; this gap is analogous to their speed,

2a. the maneuver should only be initiated if the time gap (also known as time-tocollision [35] $)^{2}$ is greater than a given value $\left(t_{\text {gap_m }}\right)$,

$2 \mathrm{~b}$. eventually (once the maneuver is finished) the vehicles should form a platoon and the velocity of all vehicles should reach $\left\|v_{\text {des }} \pm \varepsilon\right\|$, where $\varepsilon$ is a user-defined metric,

3a. the cars cannot exceed the practical velocity bounds, e.g. $v_{\min } \leq v \leq v_{\max }$, and

3b. the control inputs are bounded, i.e. $\alpha_{\min } \leq \alpha_{x} \leq \alpha_{\max }$ and $\delta_{\min } \leq \delta \leq \delta_{\max }$.

Note that these time gaps will be used for the control design part. In addition, we consider a desired platoon speed $v_{\text {des }}$ and a constant time gap $t_{\text {gap }}$. As a result, the distance between two vehicles in the platoon should not be smaller than $v_{\text {des }} \cdot t_{\text {gap }}$. During the lane change, the time gap that the merging vehicle should respect is $t_{\text {gap }} m<t_{\text {gap }}$. The numerical values of these parameters are given in Table 4 . These requirements can be encoded as reachability/safety problems, e.g. using the monitors of [10].

\footnotetext{
${ }^{1}$ By vehicle distance, we assume the difference between the longitudinal positions of two vehicles with respect to the center of gravity in their rear axles.

${ }^{2}$ Time-to-collision (TTC) corresponds to the time required for two vehicles to collide if they continue at their present speed and on the same path.
} 


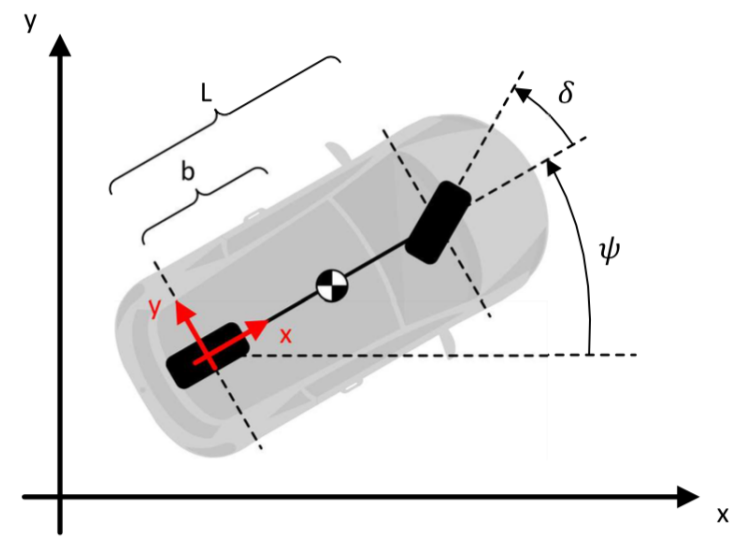

Figure 2: Bicycle Model - Physical Interpretation [20]

\section{$2.2 \quad$ Vehicle Dynamics}

For vehicle dynamics, there exists a large variety of models ranging from simpler to more complicated. Typically, the more complicated the model is, the more accurately it captures the physical behavior of the vehicle [31]. Among others, one could select from point-mass, kinematic, dynamic, and multi-body models, see e.g. [2]. In the literature of autonomous vehicles, dynamic and kinematic bicycle models are commonly used [22]. Unlike kinematic models, dynamic bicycle models consider the tire slip. Note that the standard bicycle model is also known as single-track model [31]. As for tire models, there are various alternatives from linear to nonlinear ones [29].

In this benchmark, we consider a dynamic bicycle model with a linear tire model. The selection of a dynamic model is made as the tire slip describes important phenomena, e.g. understeering. At the same time, the model is assumed linear to avoid computational complexity. The bicycle model that we consider is shown in Figure 2.

Similar to [31, Chapter 2], the state vector contains

- the longitudinal position of the rear axle $x_{r}$,

- the lateral position of the rear axle $y_{r}$,

- the yaw angle $\psi$,

- the longitudinal velocity $v_{x}$,

- the lateral velocity at the center of the rear axle $v_{y}$,

- the yaw rate $\omega$.

The model inputs are the longitudinal acceleration $a_{x}$ and the steering angle $\delta$. The state vector is completely measured and we model additive measurement noise in all state dimensions. The disturbances are defined as three normalized forces, with the error force $e_{f_{x}}^{d}$ acting in longitudinal direction, $e_{f_{y, f}}^{d}$ acting in lateral direction at the front axle and $e_{f_{y, r}}^{d}$ acting in 
Table 1: Parameters for bicycle model

\begin{tabular}{llc}
\hline Description & Symbol & Value \\
\hline wheelbase $(\mathrm{m})$ & $L$ & 2.7 \\
gravitational constant $\left(\mathrm{m} / \mathrm{s}^{2}\right)$ & $g$ & 9.81 \\
friction coefficient & $\mu$ & 0.8 \\
distance from front wheels to center of gravity & $a$ & $\left(1-\frac{b}{L}\right) \cdot L$ \\
ratio of mass to rotational inertia $\left(\mathrm{m}^{2} / \mathrm{s}^{2}\right)$ & $I_{z} / m$ & 1.57 \\
relative position of center of gravity & $b / L$ & 0.57 \\
relative front tire stiffness & $c_{f}$ & -10.8 \\
relative rear tire stiffness & $c_{r}$ & -17.8 \\
\hline
\end{tabular}

lateral direction at the rear axle. In vector form, the state vector is expressed as

$$
\begin{aligned}
& x=\left[x_{r}, y_{r}, \psi, v_{x}, v_{y}, \omega\right]^{\mathrm{T}} \\
& u=\left[a_{x}, \delta\right]^{\mathrm{T}} \\
& y=\left[x_{r}, y_{r}, \psi, v_{x}, v_{y}, \omega\right]^{\mathrm{T}} \\
& \nu=\left[e_{x_{r}}^{m}, e_{y_{r}}^{m}, e_{\psi}^{m}, e_{v_{x}}^{m}, e_{v_{y}}^{m}, e_{\omega}^{m}\right]^{\mathrm{T}} \\
& w=\left[e_{f_{x}}^{d}, e_{f_{y, f}}^{d}, e_{f_{y, r}}^{d}\right]^{\mathrm{T}} \\
& \text { state wrt. rear axle } \\
& \text { input, measured } \\
& \text { measurement } \\
& \text { meas. err. } \\
& \text { disturbance }
\end{aligned}
$$

Starting from first-principles, as shown in [31], the differential equations of the dynamic bicycle model are defined:

$$
f_{B}(x, u)=\left\{\begin{array}{l}
\dot{x}_{1}=x_{4} \cos \left(x_{3}\right)-x_{5} \sin \left(x_{3}\right) \\
\dot{x}_{2}=x_{4} \sin \left(x_{3}\right)+x_{5} \cos \left(x_{3}\right) \\
\dot{x}_{3}=x_{6} \\
\dot{x}_{4}=u_{1}+x_{5} x_{6}+w_{1} \\
\dot{x}_{5}=f_{y, f}(x, u, w)+f_{y, r}(x, w)-x_{4} x_{6} \\
\dot{x}_{6}=a \frac{m}{J}\left(f_{y, f}(x, u, w)\right)-b \frac{m}{J}\left(f_{y, r}(x, w)\right)
\end{array}\right.
$$

with the normalized front and rear lateral forces $f_{y, f}(x, u), f_{y, r}(x)$ given as

$$
\begin{aligned}
f_{y, f}(x, u) & =-c_{f} \mu g \frac{b}{a+b}\left(\frac{x_{5}+(a+b) \cdot x_{6}}{x_{4}}-u_{2}\right)+w_{2} \\
f_{y, r}(x) & =-c_{r} \mu g \frac{a}{a+b} \frac{x_{5}}{x_{4}}+w_{3} .
\end{aligned}
$$

Note that we assume a global coordinate system and the variables are described in absolute coordinates. The state of the system is often expressed in different coordinates [16], e.g. using a polar-coordinate representation of the vehicle velocity, which is defined by the slip-angle $\beta=\arctan \left(v_{y} / v_{x}\right)$ and the absolute velocity $v_{a b s}=\sqrt{v_{x}^{2}+v_{y}^{2}}$, with the direction of motion $\theta=\psi+\beta$.

The model parameters that we opt for are taken from [15] and are provided in Table 1. More details about the parameter estimation and identification can be found at [4]. 
Table 2: Maximum values of measurement errors

\begin{tabular}{cccccc}
\hline$\nu_{1}: e_{x_{r}}^{m}[m]$ & $\nu_{2}: e_{y_{r}}^{m}[m]$ & $\nu_{3}: e_{\psi}^{m}\left[{ }^{\circ}\right]$ & $\nu_{4}: e_{v_{x}}^{m}[m / s]$ & $\nu_{5}: e_{v_{y}}^{m}[m / s]$ & $\nu_{6}: e_{\omega}^{m}\left[{ }^{\circ} / s\right]$ \\
\hline 0.04 & 0.04 & 1 & 0.05 & 0.05 & 2 \\
\hline
\end{tabular}

Table 3: Maximum values of disturbances

\begin{tabular}{ccc}
\hline$w_{1}: e_{f_{x}}^{d}\left[m / s^{2}\right]$ & $w_{2}: e_{f_{y, f}}^{d}\left[m / s^{2}\right]$ & $w_{3}: e_{f_{y, r}}^{d}\left[m / s^{2}\right]$ \\
\hline 0.1 & 0.057 & 0.043 \\
\hline
\end{tabular}

The nonlinear model is linearized around a set of operating points using standard pointwise linearization, e.g. [17]. For linearization purposes, we consider $x_{o p}=[0,70 / 3.6,0,0,0,0]$. Finally, we obtain the state-space representation

$$
\begin{gathered}
y=C \cdot x+v \\
\text { where } A \approx\left[\begin{array}{cccccc}
0 & 0 & 0 & 1.0000 & 0 & 0 \\
0 & 0 & 19.4444 & 0 & 1.0000 & 0 \\
0 & 0 & 0 & 0 & 0 & 1.0000 \\
0 & 0 & 0 & 0 & 0 & 0 \\
0 & 0 & 0 & 0 & -5.5739 & -17.5748 \\
0 & 0 & 0 & 0 & 1.1909 & -6.7936
\end{array}\right], B=\left[\begin{array}{cccc}
0 & 0 \\
0 & 0 \\
0 & 0 \\
1.0000 & 0 \\
0 & 48.3123 \\
0 & 35.7265
\end{array}\right], \\
B_{d} \approx\left[\begin{array}{cccccccc}
0 & 0 & 0 \\
0 & 0 & 0 \\
0 & 0 & 0 \\
1.0000 & 0 & 0 \\
0 & 1.0000 & 1.0000 \\
0 & 0.7395 & -0.9803
\end{array}\right] \text { and } C=\left[\begin{array}{cccccc}
1 & 0 & 0 & 0 & 0 & 0 \\
0 & 1 & 0 & 0 & 0 & 0 \\
0 & 0 & 1 & 0 & 0 & 0 \\
0 & 0 & 0 & 1 & 0 & 0 \\
0 & 0 & 0 & 0 & 1 & 0 \\
0 & 0 & 0 & 0 & 0 & 1
\end{array}\right] .
\end{gathered}
$$

The maximum disturbances and maximum measurement errors are taken from [15] and are given in Tables 2 and 3. Note that the values of the $A$ and $B$ matrices that we present here are rounded. For the computations, we use the double-precision values that can be found at the associated MATLAB files. We opt for a linear model for computational reasons; such a model is amenable to efficient reachability algorithms for PWA dynamics.

\subsection{Low-level Control}

The objective of the low controller of each vehicle is to regulate its position and velocity in accordance with the behavior of the other vehicles. At the same time, the lane change maneuver should be safe. Once the maneuver is completed, the vehicle platoon should maintain the predefined vehicle speed $v_{\text {des }}$. The control scheme is shown in Figure 3.

As all the vehicles are described by the same physical model, we use the same low-level controller for all vehicles. In particular, we utilize a linear controller

$$
u=-K \cdot y=-K \cdot(x+v)
$$




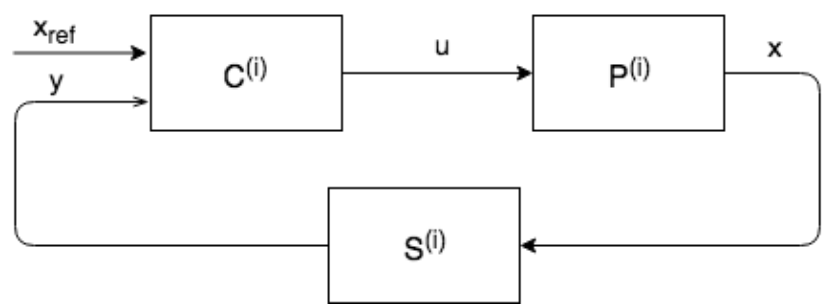

Figure 3: Low-level control loop - $C^{(i)}$ is the controller, $S^{(i)}$ is the sensor module and $P^{(i)}$ is the plant of the $i^{\text {th }}$ vehicle.

that renders the closed-loop system asymptotically stable. Opting for an LQR (Linear Quadratic Regulation) controller with weighting matrices

$$
Q=\left[\begin{array}{cccccc}
1 & 0 & 0 & 0 & 0 & 0 \\
0 & 1 & 0 & 0 & 0 & 0 \\
0 & 0 & 1 / 180 & 0 & 0 & 0 \\
0 & 0 & 0 & 5 & 0 & 0 \\
0 & 0 & 0 & 0 & 5 & 0 \\
0 & 0 & 0 & 0 & 0 & 5 / 180
\end{array}\right], R=\left[\begin{array}{cc}
1 & 0 \\
0 & 180 / \pi
\end{array}\right], \text { and } N=\mathbf{0}_{6,2}
$$

we numerically solve the continuous Algebraic Ricatti Equation with MATLAB and obtain our state-feedback matrix

$$
K=\left[\begin{array}{cccccc}
1.0000 & 0 & 0 & 2.6458 & 0 & 0 \\
0 & 0.1321 & 1.6970 & 0 & 0.0457 & 0.2829
\end{array}\right]
$$

Checking the eigenvalues of $A-B K$ matrix have $\operatorname{Re}(\operatorname{eig}(A-B K))<0$, we validate that the system is indeed stable.

\subsection{Supervisory Control}

The next step is to define the references (set points) $x_{r e f}$ for the controller of each vehicle. We employ a consensus-based cooperation scheme where the set points of each vehicle are defined by the states of its neighboring vehicles. As such, the controllers are expressed by

$$
\begin{aligned}
u & =-K\left(y-x_{r e f}\right)=-K\left(x-x_{r e f}+v\right) \text { where } \\
x_{r e f} & =\left[x_{r, r e f}, y_{r, r e f}, \psi_{r e f}, v_{x, r e f}, v_{y, r e f}, \omega_{r e f}\right] .
\end{aligned}
$$

Note that the length of a lane is assumed to be 3.5 meters. The control inputs are bounded. In particular, the acceleration remains in the range

$$
-3 \leq u_{1} \leq 2
$$

For the steering angle, we consider that

$$
-\pi / 4 \leq u_{2} \leq \pi / 4
$$

However, the reference values are different for each vehicle. 
Leader Vehicle. For the leader vehicle in the left lane (no. 3, pink in figures), we select

$$
\begin{aligned}
& v_{x, r e f}^{(3)}=\max \left(v_{\text {des }}, v_{x}^{(2)}\right) \\
& x_{r, \text { ref }}^{(3)}=\max \left(x_{r}^{(3)}, x_{r}^{(2)}+t_{\text {gap }} \cdot v_{x}^{(2)}\right) \\
& y_{r, \text { ref }}^{(3)}=0, \quad \psi_{\text {ref }}^{(3)}=0, \quad v_{y, r e f}^{(3)}=0, \text { and } \omega_{r e f}^{(3)}=0 .
\end{aligned}
$$

In essence, the leader vehicle should maintain the desired platoon velocity or accelerate when the vehicle behind it starts accelerating. Accordingly, the reference position is defined.

Rear Vehicle. For the vehicle at the tail of the platoon in the left lane (no. 1, red in figures), we select

$$
\begin{aligned}
& v_{x, r e f}^{(1)}=\min \left(v_{x}^{(2)}, v_{x}^{(4)}\right) \\
& x_{r, r e f}^{(1)}=\min \left(x_{r}^{(2)}-t_{g a p} \cdot v_{x}^{(2)}, x_{r}^{(4)}-t_{g a p} \cdot v_{x}^{(4)}\right) \\
& y_{r, \text { ref }}^{(1)}=0, \quad \psi_{r e f}^{(1)}=0, \quad v_{y, r e f}^{(1)}=0, \text { and } \omega_{r e f}^{(1)}=0 .
\end{aligned}
$$

That means that the rear vehicle should maintain the smaller speed between the merging vehicle (no. 4) and the vehicle in front of it (no. 2) to avoid any crashes.

Interior Vehicle. For the vehicle in the middle of the platoon in the left lane (no. 2, yellow in figures), we select

$$
\begin{aligned}
& v_{x, \text { ref }}^{(2)}=v_{x}^{(3)} \\
& x_{r, \text { ref }}^{(2)}=\max \left(\frac{x_{r}^{(2)}-t_{g a p} \cdot v_{x}^{(2)}+\max \left(x_{r}^{(1)}+t_{g a p} \cdot v_{x}^{(1)}, x_{r}^{(4)}+t_{g a p} \cdot v_{x}^{(4)}\right)}{2}, x_{r}^{(2)}-t_{g a p} \cdot v_{x}^{(2)}\right) \\
& y_{r, \text { ref }}^{(2)}=0, \quad \psi_{\text {ref }}^{(2)}=0, \quad v_{y, \text { ref }}^{(2)}=0, \text { and } \omega_{\text {ref }}^{(2)}=0 .
\end{aligned}
$$

That means that this vehicle should accelerate if there is not enough space for the merging vehicle to do the lane change. At the same time, it should respect the velocity speed of the leading vehicle.

Merging vehicle. For the vehicle in the right lane (no. 4, green in figures), which is doing the maneuver, we consider two phases. The first one is the pre-processing/preparation step where the vehicle needs to check if and when it is feasible to do the maneuver. In essence, the vehicle needs to regulate its velocity with respect to the platoon velocity (while guaranteeing that there is enough space margin). For that case, we choose

$$
\begin{aligned}
& v_{x, \text { ref }}^{(4)}=v_{x}^{(2)} \\
& x_{r, \text { ref }}^{(4)}=\max \left(x_{r}^{(2)}-t_{\text {gap }} \cdot v_{x}^{(2)}, \frac{x_{r}^{(2)}-t_{g a p} \cdot v_{x}^{(2)}+x_{r}^{(1)}+t_{g a p} \cdot v_{x}^{(1)}}{2}\right) \\
& \boldsymbol{y}_{r, \text { ref }}^{(4)}=\mathbf{0}, \quad \psi_{\text {ref }}^{(4)}=0, \quad v_{y, \text { ref }}^{(4)}=0, \text { and } \omega_{r e f}^{(4)}=0 .
\end{aligned}
$$




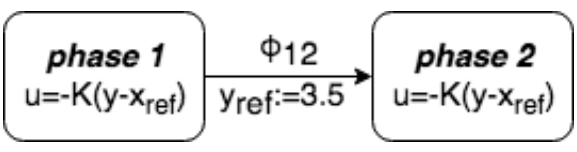

Figure 4: Schematic of lane change maneuver - 2 phases of merging vehicle movement

Table 4: Parameters for control design

\begin{tabular}{llc}
\hline Description & Symbol & Value \\
\hline minimum velocity $(\mathrm{km} / \mathrm{h})$ & $v_{\min }$ & 0 \\
maximum velocity $(\mathrm{km} / \mathrm{h})$ & $v_{\max }$ & 150 \\
minimum acceleration $\left(\mathrm{m} / \mathrm{s}^{2}\right)$ & $\alpha_{\min }$ & -3 \\
maximum acceleration $\left(\mathrm{m} / \mathrm{s}^{2}\right)$ & $\alpha_{\max }$ & 2 \\
minimum steering angle $(\mathrm{rad})$ & $\delta_{\min }$ & $-\pi / 4$ \\
maximum steering angle $(\mathrm{rad})$ & $\delta_{\max }$ & $\pi / 4$ \\
desired platoon vehicle $(\mathrm{km} / \mathrm{h})$ & $v_{\text {des }}$ & 70 \\
time gap (sec.) & $t_{\text {gap }}$ & 1.5 \\
minimum time gap $(\mathrm{sec})$. & $t_{\text {gap_m }}$ & 1 \\
constant gap $(\mathrm{m})$ & gap & $\frac{70}{3.6} * 1.5$ \\
\hline
\end{tabular}

In the second phase, the vehicle starts the merging maneuver. This practically means that its lateral position should change. To do so, the lateral position reference $y_{r, r e f}$ should be modified.

$$
\begin{aligned}
& v_{x, \text { ref }}^{(4)}=v_{x}^{(2)} \\
& x_{r, \text { ref }}^{(4)}=\max \left(x_{r}^{(2)}-t_{g a p} \cdot v_{x}^{(2)}, \frac{x_{r}^{(2)}-t_{g a p} \cdot v_{x}^{(2)}+x_{r}^{(1)}+t_{g a p} \cdot v_{x}^{(1)}}{2}\right) \\
& \boldsymbol{y}_{r, \text { ref }}^{(4)}=\mathbf{3 . 5}, \quad \psi_{r e f}^{(4)}=0, \quad v_{y, r e f}^{(4)}=0, \text { and } \omega_{r e f}^{(4)}=0 .
\end{aligned}
$$

The condition that should be valid to initiate a safe lane change (transition from phase 1 to 2 ) is

$$
\phi_{12}:=\left\{x_{r}^{(4)}<x_{r}^{(2)}-t_{\text {gap } \_m} \cdot v_{x}^{(2)} \text { and } x_{r}^{(4)}>x_{r}^{(1)}+t_{\text {gap_m } m} \cdot v_{x}^{(1)}\right\} .
$$

The switching logic can be visualized in Figure 4. The parameter values and initial conditions used for simulation purposes are shown in Tables 4 and 5 respectively.

\section{Simulation Results}

The simulation results for the specified scenario are depicted in Figure 5. The 2D images have been constructed using the plot and patch commands of MATLAB. Figure 6 shows the position of the vehicle doing the lane change and the evolution of all vehicles velocity over time. For the 3D images, we have used Unity game engine [6]. After setting up the scenario in Unity, we simulate the lane change maneuver and get a $3 \mathrm{D}$ video [12]. Figure 7 illustrates the main instances of the maneuver and it is obtained through Unity image capture. 
Table 5: Initial conditions of each vehicle

\begin{tabular}{llllllll}
\hline Vehicle & $i$ & $x_{1}^{(i)}(0)$ & $x_{2}^{(i)}(0)$ & $x_{3}^{(i)}(0)$ & $x_{4}^{(i)}(0)$ & $x_{5}^{(i)}(0)$ & $x_{6}^{(i)}(0)$ \\
\hline Rear & 1 & 0 & 3.5 & 0 & $v_{\text {des }}$ & 0 & 0 \\
Interior & 2 & gap & 3.5 & 0 & $v_{\text {des }}$ & 0 & 0 \\
Leader & 3 & $2 *$ gap & 3.5 & 0 & $v_{\text {des }}$ & 0 & 0 \\
Merging & 4 & $2 *$ gap & 0 & 0 & $v_{\text {des }} / 2$ & 0 & 0 \\
\hline
\end{tabular}

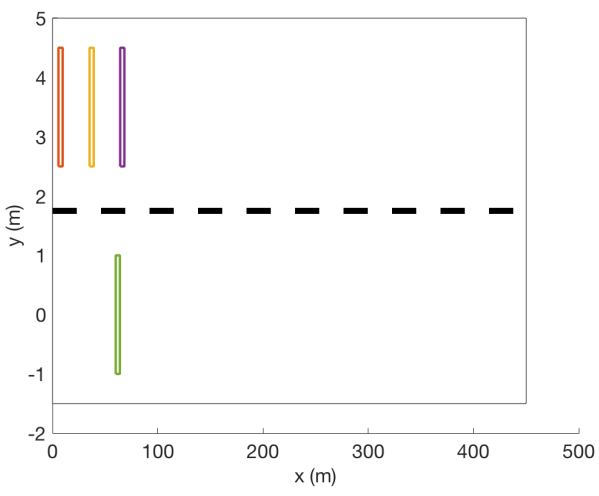

(a) Initial Phase (before maneuver)

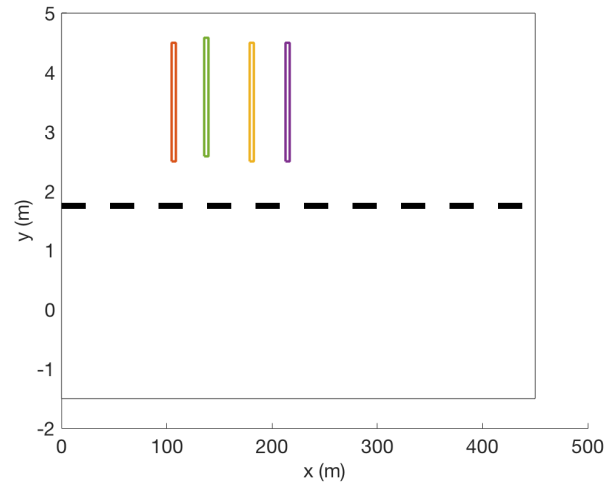

(c) Maneuver just completed

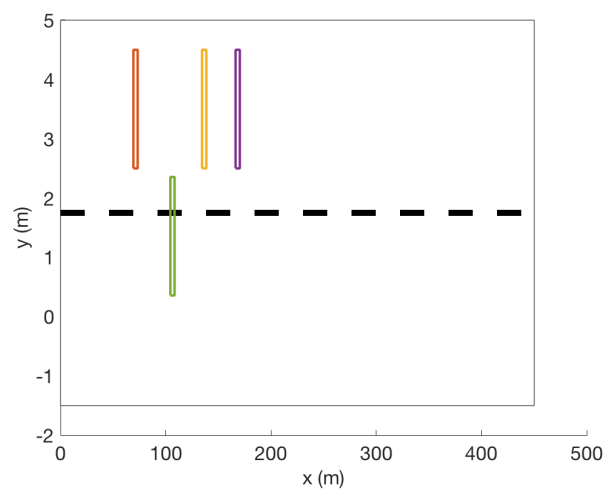

(b) Maneuver initiated

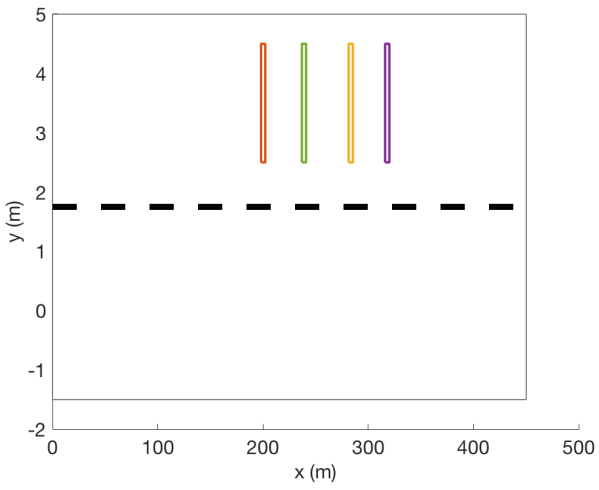

(d) Maneuver finished and platoon stabilized

Figure 5: Graphical illustration of a lane change maneuver - Simulation results with MATLAB 


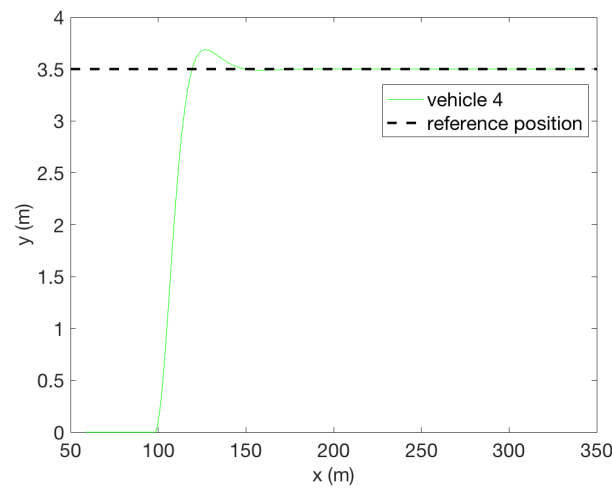

(a) Evolution of maneuvering vehicle position

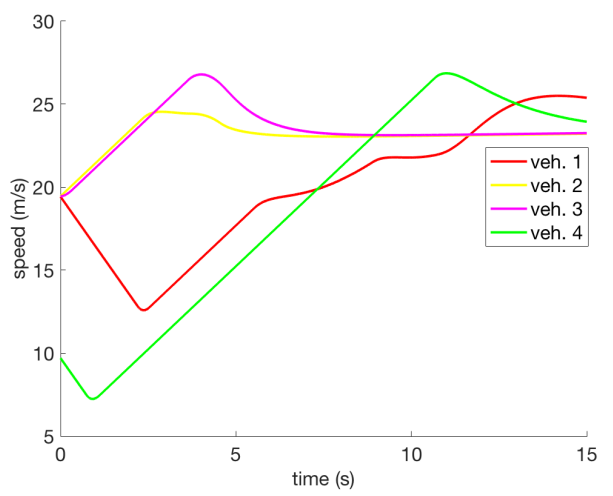

(b) Evolution of vehicles velocities over time

Figure 6: Graphical illustration of a lane change maneuver - Simulation results with MATLAB

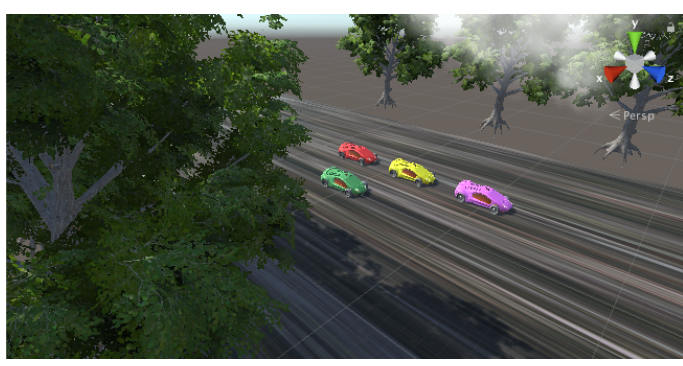

(a) Initial Phase (before maneuver)

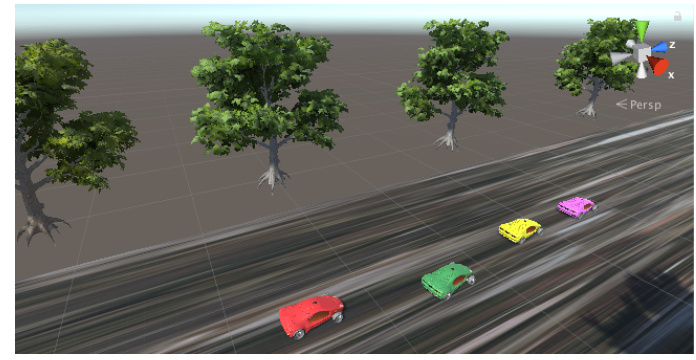

(c) Maneuver - in progress

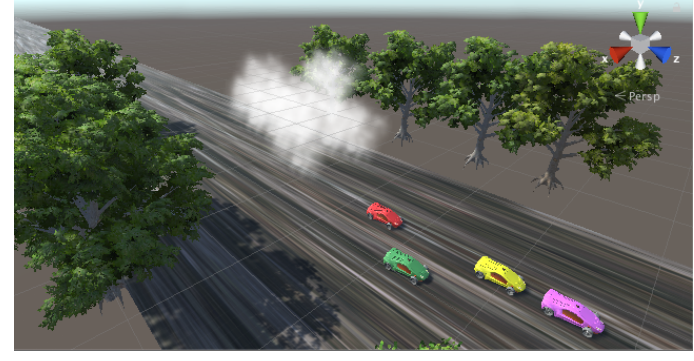

(b) Maneuver has begun

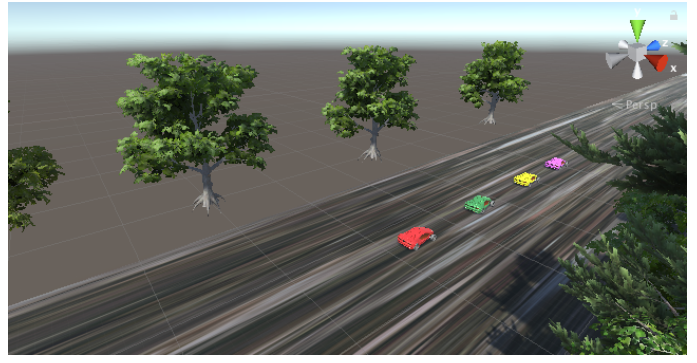

(d) Maneuver finished and platoon stabilized

Figure 7: Graphical illustration of a lane change maneuver - Simulation results with Unity

\section{Outlook}

Autonomous vehicles have stimulated both industrial and academic interest, and have induced major advances in several research areas, such as perception, sensing, control theory, verification, and testing. Verification efforts have been directed towards the validation of vehicle modules and subsystems, such as cruise control and lane-keeping. 
The current model can serve as a basis for lane change maneuvers of autonomous vehicles tailored for automated verification tools. It is not very complicated but still realistic and expressive. Disturbances and measurement noise increase the computational efforts during analysis with standard simulation tools. The benchmark can be extended in several directions. Different controllers and vehicle models could be straightforwardly used and analysed.

\section{References}

[1] Matthias Althoff and John M Dolan. Set-based computation of vehicle behaviors for the online verification of autonomous vehicles. In ITSC Conf., 2011.

[2] Matthias Althoff, Markus Koschi, and Stefanie Manzinger. CommonRoad: Composable benchmarks for motion planning on roads. In IV Symposium, 2017.

[3] Vadim A Butakov and Petros Ioannou. Personalized driver/vehicle lane change models for adas. IEEE Transactions on Vehicular Technology, 64(10):4422-4431, 2015.

[4] Javier Sanchez Cubillo, Simone Schuler, Daniel Heß, Maria Prandini, and Mark Burgin. UnCoVerCPS Deliverable D5.1: Report on Application Models. https://cps-vo.org/node/24201, 2015.

[5] Dmitri Dolgov, Sebastian Thrun, Michael Montemerlo, and James Diebel. Path planning for autonomous vehicles in unknown semi-structured environments. The International Journal of Robotics Research, 29(5):485-501, 2010.

[6] Unity Game Engine. Official website. http://unity3d. com, 2008.

[7] Daniel J Fagnant and Kara Kockelman. Preparing a nation for autonomous vehicles: opportunities, barriers and policy recommendations. Transportation Research Part A: Policy and Practice, $77: 167-181,2015$.

[8] Paolo Falcone, Francesco Borrelli, Jahan Asgari, Hongtei Eric Tseng, and Davor Hrovat. Predictive active steering control for autonomous vehicle systems. IEEE Transactions on control systems technology, 2007.

[9] Emilio Frazzoli. Robust hybrid control for autonomous vehicle motion planning. PhD thesis, 2001.

[10] Goran Frehse, Nikolaos Kekatos, Dejan Nickovic, Jens Oehlerking, Simone Schuler, Alexander Walsch, and Matthias Woerhle. A toolchain for verifying safety properties of hybrid automata via pattern templates. In ACC Conference, 2018.

[11] Yiqi Gao, Andrew Gray, H. Eric Tseng, and Francesco Borrelli. A tube-based robust nonlinear predictive control approach to semiautonomous ground vehicles. Vehicle System Dynamics, 2014.

[12] Deepthi Gopalakrishna. Simulation of autonomous vehicles with Unity. https://github.com/ deepkrishna/AV-simulation, 2018.

[13] Andrew Gray, Yiqi Gao, J Karl Hedrick, and Francesco Borrelli. Robust predictive control for semi-autonomous vehicles with an uncertain driver model. In IV Symposium (IV), 2013.

[14] Feng Guo, Brian M Wotring, and Jonathan F Antin. Evaluation of lane change collision avoidance systems using the national advanced driving simulator. Technical report, 2010.

[15] Marianne Hartung, Daniel Heß, Ray Lattarulo, Jens Oehlerking, Joshué Pérez, and Alexander Rausch. UnCoVerCPS Deliverable D5.2: Report on Conformance Testing of Application Models. https://cps-vo.org/node/39012, 2016.

[16] Daniel Heß, Matthias Althoff, and Thomas Sattel. Formal verification of maneuver automata for parameterized motion primitives. In IROS Conf., 2014.

[17] Daniel Heß, Bastian Schürmann, Marcelo Forets, and Goran Frehse. UnCoVerCPS Deliverable D3.2: Report on Precomputation of Reachable Sets and Advances in Reachability Analysis. https: //cps-vo.org/node/39010, 2017.

[18] ML Ho, PT Chan, and AB Rad. Lane change algorithm for autonomous vehicles via virtual curvature method. Journal of Advanced Transportation, 43(1):47-70, 2009. 
[19] Jonas Jansson and Fredrik Gustafsson. A framework and automotive application of collision avoidance decision making. 2008.

[20] Michael Kaiser. Untersuchung zur online abgesicherten Fahrer-Fahrzeug Interaktion in Kollisionsvermeidungs-Szenarien. PhD thesis, Technische Universität Braunschweig, 2017.

[21] Uwe Kiencke and Lars Nielsen. Automotive control systems: for engine, driveline, and vehicle. Springer Science \& Business Media, 2005.

[22] Jason Kong, Mark Pfeiffer, Georg Schildbach, and Francesco Borrelli. Kinematic and dynamic vehicle models for autonomous driving control design. In IV Symposium, 2015.

[23] Markus Koschi and Matthias Althoff. Spot: A tool for set-based prediction of traffic participants. In IV Symposium. IEEE, 2017.

[24] Sarah M Loos, André Platzer, and Ligia Nistor. Adaptive cruise control: Hybrid, distributed, and now formally verified. In International Symposium on Formal Methods, 2011.

[25] Xiao-Yun Lu and J Karl Hedrick. Longitudinal control algorithm for automated vehicle merging. International Journal of Control, 2003.

[26] Silvia Magdici and Matthias Althoff. Fail-safe motion planning of autonomous vehicles. In ITSC Conf., 2016.

[27] Silvia Magdici, Zhenzhang Ye, and Matthias Althoff. Determining the maximum time horizon for vehicles to safely follow a trajectory. In ITSC Conf., 2017.

[28] Julia Nilsson, Mattias Brännström, Erik Coelingh, and Jonas Fredriksson. Lane change maneuvers for automated vehicles. ITS, 2017.

[29] Hans Pacejka. Tire and vehicle dynamics. Elsevier, 2005.

[30] Zhihua Qu. Cooperative control of dynamical systems: applications to autonomous vehicles. Springer Science \& Business Media, 2009.

[31] Rajesh Rajamani. Vehicle dynamics and control. Springer Science \& Business Media, 2011.

[32] Bastian Schürmann and Matthias Althoff. Convex interpolation control with formal guarantees for disturbed and constrained nonlinear systems. In HSCC, 2017.

[33] Martin Treiber, Arne Kesting, and Dirk Helbing. Understanding widely scattered traffic flows, the capacity drop, and platoons as effects of variance-driven time gaps. Physical review E, 2006.

[34] Torgeir Vaa, Merja Penttinen, and I Spyropoulou. Intelligent transport systems and effects on road traffic accidents: state of the art. IET Intelligent Transport Systems, 1(2):81-88, 2007.

[35] Richard van der Horst and Jeroen Hogema. Time-to-collision and collision avoidance systems. 1993. 